\title{
Perçage d'empilages multi-materiaux
}

\author{
Sébastien Gouleau ${ }^{1, a}$, Sébastien Garnier ${ }^{2}$ et Benoît Furet ${ }^{2}$ \\ 1 SYNERVIA, 2 avenue du Professeur Jean Rouxel, 44475 Carquefou, France \\ 2 IRCCYN, UMR CNRS 6597, École Centrale, École des Mines, Université, IUT de Nantes, 1 rue de la Noë, \\ 44321 Nantes, France
}

Reçu le 15 mars 2007, accepté le 6 juillet 2007

\begin{abstract}
Résumé - La part des matériaux composites dans les structures aéronautiques devient de plus en plus importante et devrait se situer aux alentours de $50 \%$ d'ici 2012. Dans la phase d'assemblage des tronçons d'avions, les centres de production aéronautiques actuels utilisent des Unités de Perçage Automatique (UPA) pneumatiques positionnés manuellement sur un outillage dédié pour le perçage axial des panneaux de structure. Les panneaux à percer sont des assemblages de matériaux composites, aluminium et titane. En particulier, le perçage de matériaux composites (hétérogène et anisotrope) peut engendrer l'apparition de défauts, tels que le délaminage ou l'écaillage, influençant directement les propriétés mécaniques. Les paramètres de coupe caractéristiques et l'usure des outils nécessitent d'être suivis et maîtrisés pour diminuer l'apparition de ces défauts macro et microgéométriques. Cet article présente donc la problématique du perçage de panneaux multi-matériaux, en rappelant les spécificités des matériaux usinés, les moyens de générations actuels ainsi que les modes de dégradations rencontrés. Un point sera fait sur l'influence des paramètres d'usinage sur la qualité géométrique et les défauts de surface. Les conclusions montreront les résultats obtenus et les développements à mener dans un futur proche.
\end{abstract}

Mots clés : Usinage des composites / surveillance en perçage / mesure d'efforts

\begin{abstract}
A study on the drilling of composite and aluminium stocks. Composite in aeronautical structures become increasingly important and should be at the neighbourhoods of $50 \%$ between now and 2012. During the assembly, the aeronautical centers of production use pneumatic Units of Automatic Drilling manually positioned on dedicated tools for the axial drilling of the panels of structure (assemblies of composite materials, aluminium and titanium). In particular, the drilling of composites (heterogeneous and anisotropic) can generate the appearance of defects, such as delamination or flaking, influencing directly the mechanical properties. The cut parameters and tools wear requires being followed and controlled to decrease the appearance of these macro and microgeometrical defects. This article presents the problems of multi-materials parts drilling by pointing out the specificities of machining such materials, the actual generations means and all the degradations modes met during the study. A point will be made on the influence of the parameters of machining on geometrical quality and the defects of surface. The conclusions will show the results obtained and the developments to be carried out in an immediate future.
\end{abstract}

Key words: Composite drilling / supervision of drilling / strength measurement

\section{Introduction}

L'attrait dans la mécanique et dans l'aéronautique [1] de l'utilisation des matériaux composites vient du fait que l'on conçoit un composite en fonction du type d'application donc du type de chargement, ce qui est différent des matériaux classiques où on adapte la conception d'une structure en fonction du matériau constitutif.

\footnotetext{
a Auteur pour correspondance :

sebastien.gouleau@univ-nantes.fr
}

Dans ce cadre, deux types de composites sont à distinguer : les composites Grande Diffusion (GD) et les composites Haute Performance (HP). Les composites GD, représentent $95 \%$ des matériaux composites utilisés. En général, ce sont des plastiques armés ou des plastiques renforcés dont le taux volumique de renfort avoisine $30 \%$. Les principaux constituants de base sont les résines polyesters avec des fibres de verre. Les composites HP sont principalement utilisés dans l'aéronautique. Les renforts utilisés sont plutôt des fibres longues (taux de renfort 
supérieur à $50 \%$ ). Contrairement aux composites GD, leurs propriétés mécaniques, résistance mécanique et rigidité, sont à masses égales largement supérieures à celles des métaux. Dans cette étude, nous nous intéressons à l'usinage par perçage de panneaux de structure composés de composites HP en particulier HR (haute résistance mécanique) de type thermodurcissable à fibres de carbone et d'alliages d'aluminium de la série 7000 .

De nombreuses études ont été menées [2-4] sur le perçage de matériaux aéronautiques (composites, d'alliages d'aluminium et de titane) et la majorité d'entre elles mettent en évidence l'influence des actions mécaniques et thermiques sur l'apparition de défauts macro géométriques. Par contre, très peu de travaux se sont intéressés à l'optimisation du perçage de panneaux multi matériaux. Il apparaît pourtant $[5,6]$ que le perçage de panneaux multi-matériaux constitués de matériaux de caractéristiques différentes pose souvent problèmes. Par exemple, l'augmentation de la température à l'interface des panneaux composites et titane peut devenir néfaste à l'intégrité du panneaux de structure si les paramètres opératoires ne sont pas correctement maîtrisés.

De manière globale, nous cherchons à comprendre, mesurer et quantifier les actions mécaniques (poussée et couple) mises en jeu lors de la coupe du carbone et de l'aluminium. Un second objectif est d'analyser les influences des matériaux, des géométries des outils et des conditions de coupe actuellement utilisées sur la qualité et l'intégrité des surfaces produites.

\section{2 État des lieux}

\subsection{Le procédé}

Les pièces à assembler par rivetage sont souvent de matériaux différents (composites carbone, alliage d'aluminium...). Les techniques de perçage utilisées sont de deux types. Le premier type de perçage est le perçage par interpolation hélicoïdale sur machines spéciales avec des fraises PCD (diamant polycristalin) et le second type (pour la majorité des cas) est le perçage axial automatique sur machines pneumatiques portables. Ces unités de perçage sont positionnées manuellement sur un outillage dédié et utilisent des outils de type « foret $3 / 3$ » à plaquettes PCD brasées.

Persson et al. [7] ont étudié l'influence du type de perçage sur la tenue en statique et en fatigue de stratifiés carbone/époxyde $[(+45 /-45 / 0 / 90) 3]_{24 S}$. Les essais utilisés sont des essais de traction par goupille sur éprouvettes percées (résistance au matage) et de compression globale. Le tableau (Tab. 1) synthétise les résultats pour les essais de matage en quasi-statique.

Il ressort de cette étude que l'utilisation d'un foret $\mathrm{PCD}$ en perçage classique diminue d'environ $10 \%$ la contrainte à rupture de matage par rapport au détourage ou bien à l'utilisation d'un foret hélicoïdal en carbure. Cependant, aucun résultat n'est apporté quant à l'influence de l'outil utilisé sur la qualité intrinsèque des surfaces produites en lien avec les essais de matage.
Tableau 1. Influence du type de perçage sur la contrainte de matage quasi-statique.

\begin{tabular}{lccc}
\hline Méthode de perçage & $\begin{array}{c}\text { Contrainte } \\
\text { de matage } \\
(\mathrm{MPa})\end{array}$ & $\begin{array}{c}\text { Coefficient } \\
\text { de variation } \\
(\%)\end{array}$ & $\begin{array}{c}\text { Ecart par rapport } \\
\text { au meilleur } \\
(\%)\end{array}$ \\
\hline $\begin{array}{c}\text { Détourage avec fraise } \\
\text { de faible diamètre }\end{array}$ & 723 & 2,3 & - \\
Foret PCD neuf & 646 & 8,1 & $-10,7$ \\
Foret PCD usé & 642 & 2,9 & $-11,2$ \\
Foret hélicoïdal neuf & 711 & 1,0 & $-1,7$ \\
Foret hélicö̈dal usé & 702 & 1,0 & $-2,9$ \\
\hline
\end{tabular}
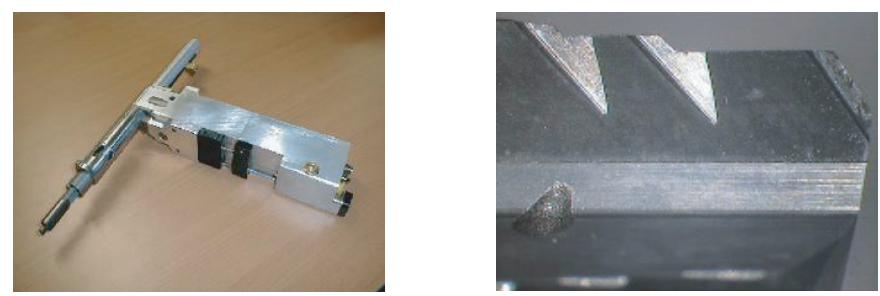

Fig. 1. Machine portable et « foret $3 / 3$ » à plaquettes $\mathrm{PCD}$ brasées.

\subsection{Les outils utilisés}

Les assemblages multi matériaux présentent des conditions de coupe antagonistes. Par exemple, dans le cas d'un assemblage carbone-titane, le carbone s'usine à vitesse élevée à l'aide d'outils PCD mais les revêtements à base de ces polycristaux ne résiste pas à l'usinage du titane. À l'inverse les carbures revêtus qui composent les outils de coupe du titane, utilisés à faible vitesse, sont usés et abrasés par le carbone.

Dans notre étude, nous avons privilégié les outils à plaquette PCD brasée sur un corps en carbure (forets $3 / 3$ ). Ces outils sont exploités à l'assemblage en production à l'aide de machine portative (Fig. 1).

\subsection{Les dégradations observées}

En raison de l'hétérogénéité et de l'anisotropie, l'usinage des composites peut engendrer des endommagements influençant directement les propriétés mécaniques contrairement aux matériaux métalliques. Les actions mécaniques mises en jeu lors de la coupe peuvent engendrer des phénomènes de « décollement» des couches de fibres appelés «plis» entre elles. Sur la figure 2 sont présentés différents types de défauts. Si ce décollement est situé au cœur de l'épaisseur de la pièce, le défaut est appelé délaminage, lorsqu'il se produit en surface pour les derniers plis, il est appelé écaillage. On peut aussi constater en usinage, des fibres non coupées sur le premier ou le dernier pli, ce défaut est dû à la nature du tissage et aux conditions opératoires.

Ghidossi et al. [8] ont montré que la coupe dégrade le composite par des mécanismes bien identifiés : délaminage, microfissuration, dégradation thermique, arrachement de fibres. Ces endommagements n'influencent 


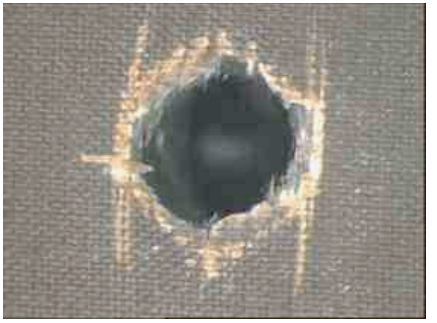

Ecaillage en perçage

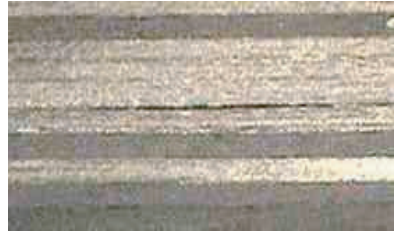

Délaminage

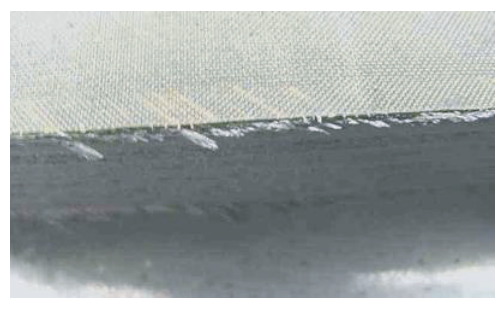

Ecaillage en fraisage

Fig. 2. Différents type de défauts.
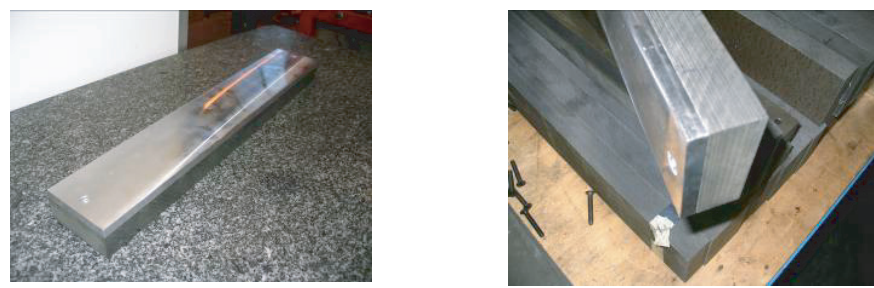

Fig. 3. Éprouvette aluminium/carbone vierge de perçage.

pas toujours le comportement mécanique des structures sollicitées, cela dépend du mode de sollicitation. Si la rupture a lieu loin des zones usinées ou si elle s'initie en compression (fermeture des fissures), les effets sont négligeables. Si la rupture s'initie au niveau des surfaces coupées, l'influence est significative, surtout si la rupture s'opère en traction transverse ou cisaillement. Dans notre cas, les éléments percés sont des panneaux de structure assemblés les uns aux autres par rivetage. La sollicitation mécanique la plus courante est donc une sollicitation de compression.

Néanmoins, afin de pouvoir diminuer et supprimer ces créations de défauts lors de l'usinage, il est nécessaire de connaître et de maîtriser les actions mises en jeu lors de la coupe à l'outil PCD dans les composites carbones.

\section{Nos expérimentations}

\subsection{Contexte expérimental}

\subsubsection{Présentation des assemblages multi matériaux}

Les assemblages concernés par cette étude sont du type carbone/aluminium (Fig. 3).

L'intérêt d'associer l'aluminium aux composites est que ces deux matériaux vont compenser mutuellement leurs défauts. Les composites sont très résistants à la charge, mais ont une faible résistance à l'impact, ainsi que de faibles propriétés statiques en cas de fissuration. L'aluminium est moins résistant à la charge, mais résiste mieux aux impacts, et à une plus grande zone plastique.
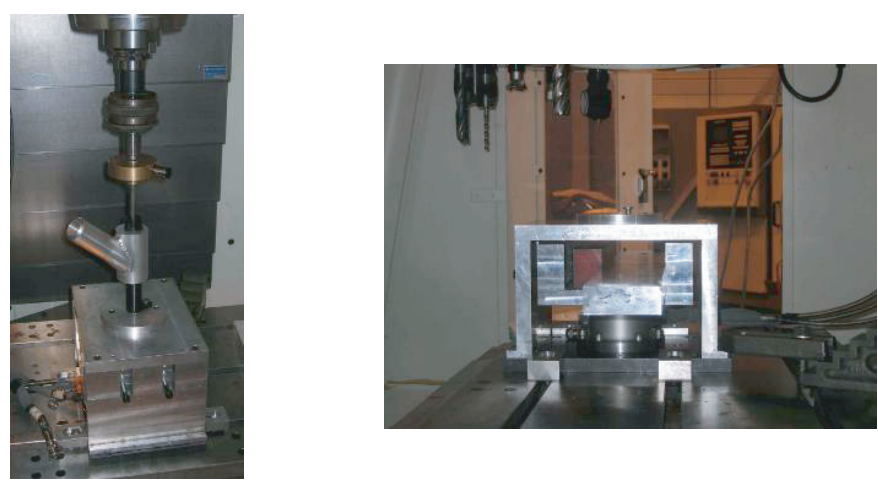

Fig. 4. Montage flottant du foret et guidage par canon de perçage.
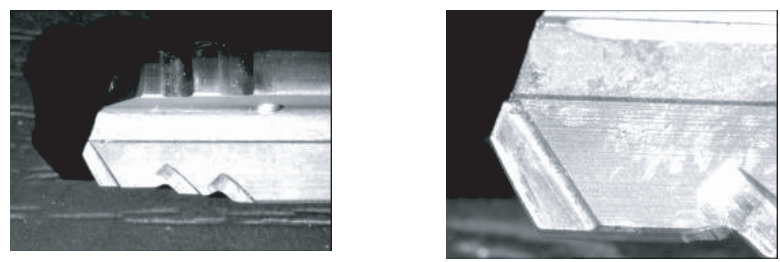

Fig. 5. Foret $3 / 3$ étagé de $\varnothing 11,1$.

\subsubsection{Présentation des moyens expérimentaux mis en œuvre}

Les essais de perçages ont été réalisés sur un centre d'usinage Hermle (Fig. 4) de la plateforme UGV de l'IUT de Nantes. L'utilisation d'un centre d'usinage nous permet de maîtriser les paramètres opératoires (vitesse de coupe et avance du foret) lors des différents essais de perçage. L'éprouvette est mise en position sur un montage spécifique de type étau. Ce montage est lié à une platine dynamométrique (platine Kistler (Fz, Mz)). L'outil est monté flottant dans le porte outil et est guidé à l'aide d'un canon de perçage ce qui permet de se rapprocher au plus près des conditions industrielles.

L'outil utilisé est un foret $3 / 3$ étagé (Fig. 5) monté flottant dans le porte-outil. Lors du perçage, il est guidé par un canon de perçage positionné au-dessus et au centre de la platine d'acquisition. L'opération de perçage s'effectue donc sans pointage préalable de l'éprouvette.

Lors de l'usinage, les copeaux (poussière de carbone et aluminium) sont aspirés. 


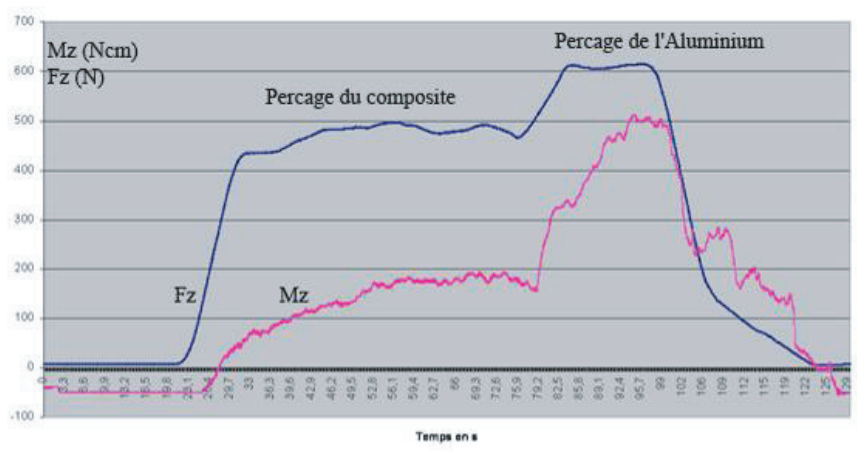

Fig. 6. Signal observé.
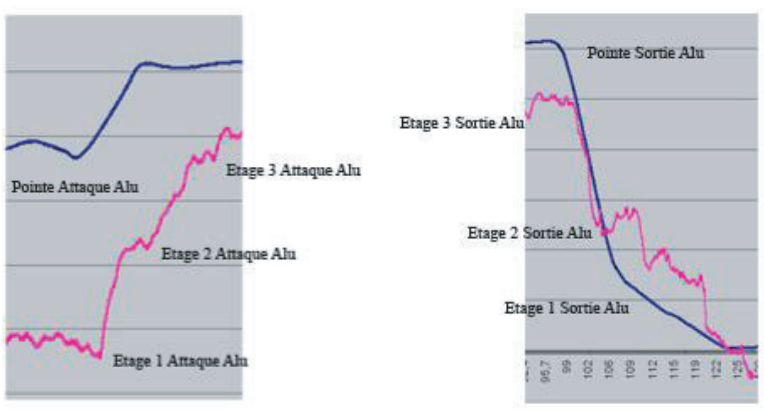

Fig. 7. Détection des signaux d'entrée et de sorties matière.

Un système de micro lubrification centre outil de la société VOGEL est utilisé afin de réduire le frottement outil/pièce.

\subsubsection{Les signaux d'efforts et de couples}

La figure 6 représente le signal observé lors du perçage d'un assemblage carbone/aluminium à iso conditions de coupe. Sur cette figure, on distingue nettement le passage du perçage du panneau composite au perçage du panneau aluminium.

La figure 7 permet de mettre en évidence les entrées et sorties matières ainsi que le passage des différents étages du foret $3 / 3$. La connaissance de ces temps de passage d'un matériau à un autre devrait nous permettre d'asservir les efforts à la nature du matériau percé.

Dans notre étude, nous cherchons à homogénéiser les charges machines (poussées et couples) tout au long du perçage de l'assemblage carbone/aluminium et de les rendre le plus faible possible. Les puissances nécessaires doivent restées admissibles par une machine de perçage portative (Fig. 1).

\subsubsection{Conditions d'essais}

Dans une première approche, les conditions expérimentales choisies pour le composite sont tablées sur des conditions industrielles. Elles sont de trois niveaux pour la vitesse de rotation ainsi que pour l'avance. Pour l'aluminium, quatre niveaux de vitesse de rotation ainsi que
Tableau 2. Paramètres expérimentaux.

\begin{tabular}{l|cc}
\hline & $\begin{array}{c}\text { Vitesse de rotation } \\
N\left(\text { tr.min }^{-1}\right)\end{array}$ & $\begin{array}{c}\text { Avance par tour } \\
f\left(\mathrm{~mm}_{\mathrm{tr}}{ }^{-1}\right)\end{array}$ \\
\hline Aluminium & 2000 & 0,02 \\
& 2200 & 0,04 \\
& 2400 & 0,06 \\
Carbone & 2600 & \\
& 1800 & 0,05 \\
& 2000 & 0,1 \\
& 2200 & 0,15 \\
\hline
\end{tabular}

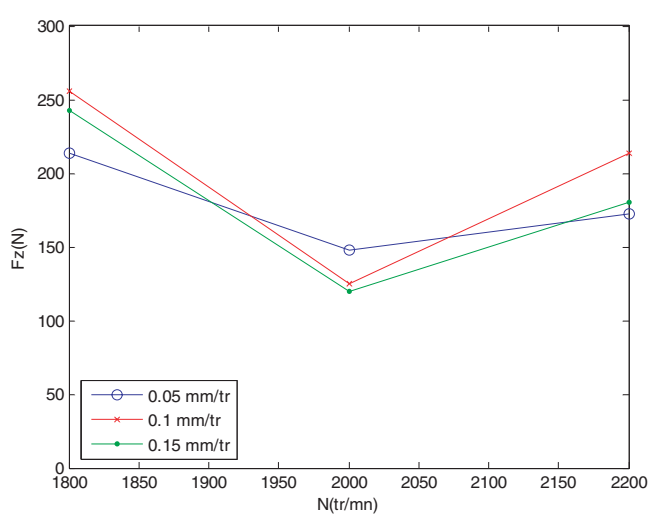

Fig. 8. Influence de la vitesse de rotation sur la poussée dans le cas de l'usinage du carbone.

trois pour l'avance ont été expérimentés. Les paramètres sont répertoriés dans le tableau 2.

\subsection{Analyse des résultats expérimentaux}

L'analyse des signaux d'efforts nous a permis d'extraire les valeurs maximales des efforts lors du perçage des éprouvettes en carbone/aluminium. Nous avons choisi d'extraire les extremums des signaux d'efforts et non leurs valeurs moyennes. En effet, si l'on arrive à minimiser la poussée et le couple, nous réussirons à minimiser le risque d'endommager la structure lors du perçage. Ces extremums sont répertoriés dans les graphiques suivants (Figs. 8 à 15).

\subsubsection{Signaux de poussée}

L'analyse des signaux d'efforts semblerait indiquer que l'influence de la vitesse de rotation sur la poussée apparaît significative aux alentours de 2000 tr.min ${ }^{-1}$ dans le cas du carbone (Fig. 8) alors qu'elle n'a pas d'effet notable dans le cas de l'aluminium (Fig. 10).

Les variations de section de copeaux (augmentation de la vitesse d'avance) n'ont pas d'influence sur la poussée dans le cas du carbone (Fig. 9). À l'inverse, dans le cas de l'aluminium (Fig. 11) la variation de section de copeaux a une influence notable sur la poussée (augmentation de l'effort de $75 \%$ ). Nous pouvons aussi mettre en relief que la poussée est deux fois plus grande dans l'aluminium que dans le carbone pour des paramètres de coupe identiques (2000 tr.min ${ }^{-1}, 0,04$ mm.tr ${ }^{-1}$ ). 


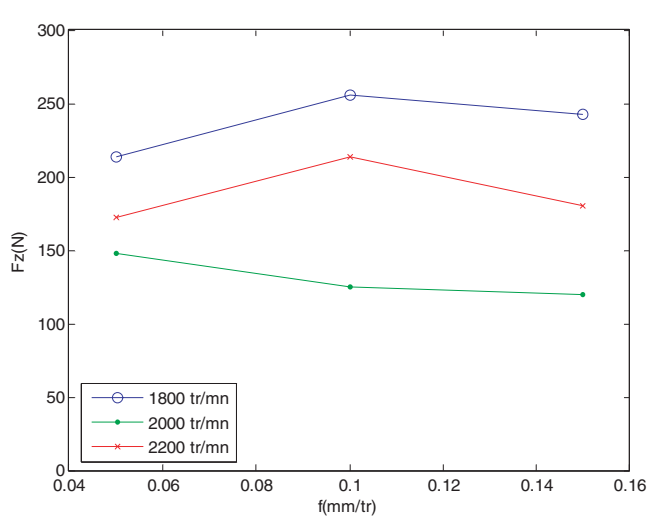

Fig. 9. Influence de l'avance sur la poussée dans le cas de l'usinage du carbone.

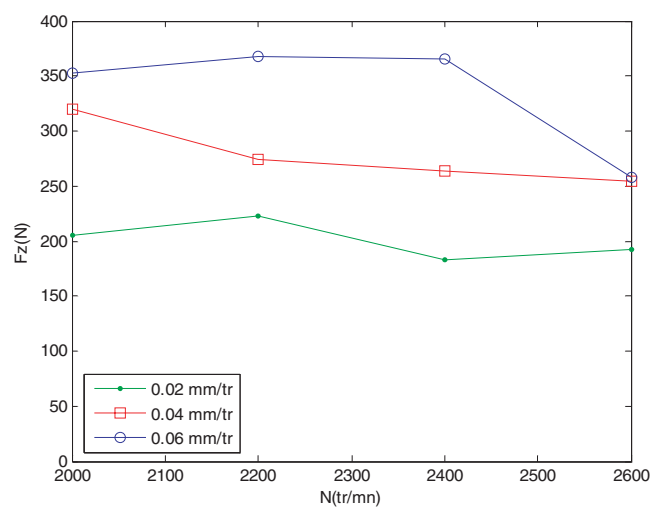

Fig. 10. Influence de la vitesse de rotation sur la poussée dans le cas de l'usinage de l'aluminium.

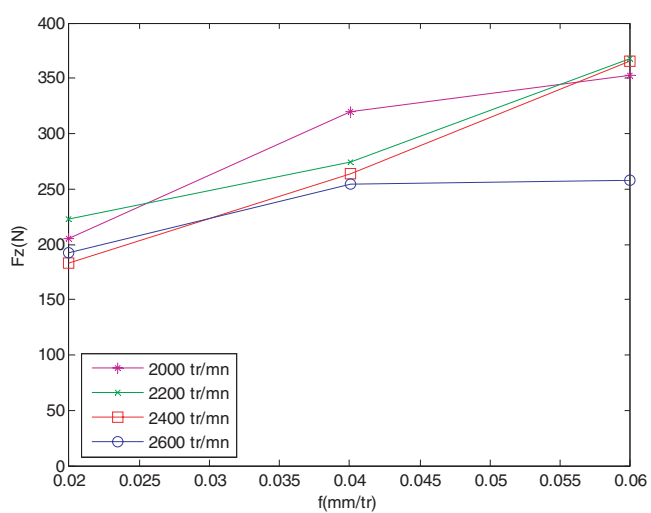

Fig. 11. Influence de l'avance sur la poussée dans le cas de l'usinage de l'aluminium.

\subsubsection{Signaux de couple}

L'analyse des signaux de couple montre que l'augmentation de la vitesse de rotation dans les plages expérimentées a peu d'influence sur le couple (Figs. 12 et 14). Par contre, on remarque que l'augmentation de la vitesse d'avance influe de façon significative sur le couple aussi bien dans l'aluminium que dans le carbone (Figs. 13 et 15). De manière identique à la poussée, nous pouvons mettre en relief que le couple est deux fois plus grand dans

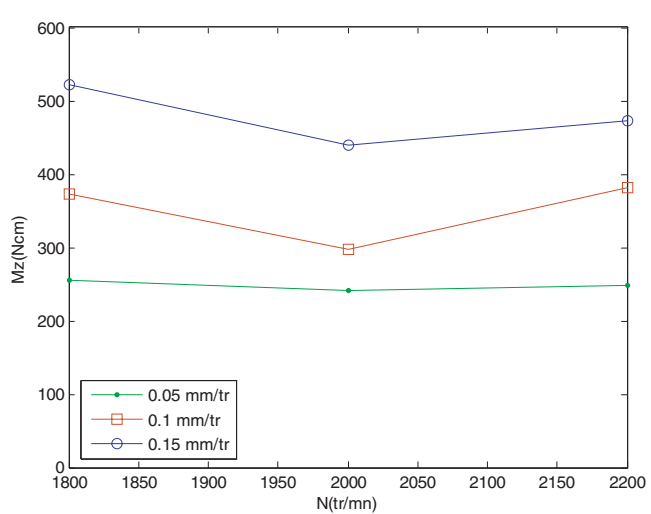

Fig. 12. Influence de la vitesse de rotation sur le couple dans le cas de l'usinage du carbone.

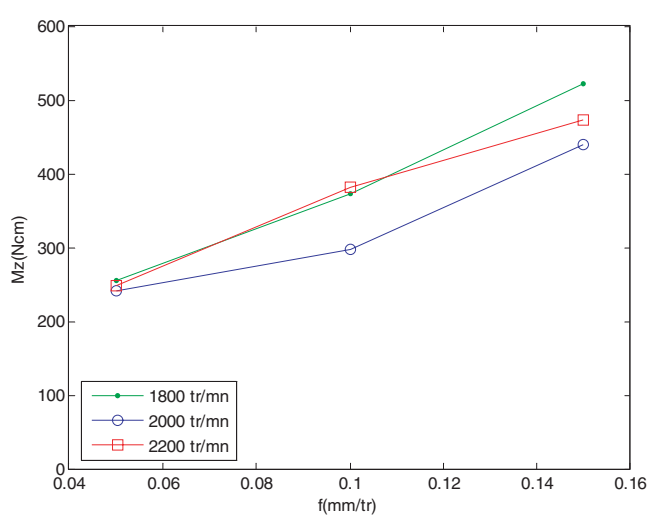

Fig. 13. Influence de l'avance sur le couple dans le cas de l'usinage du carbone.

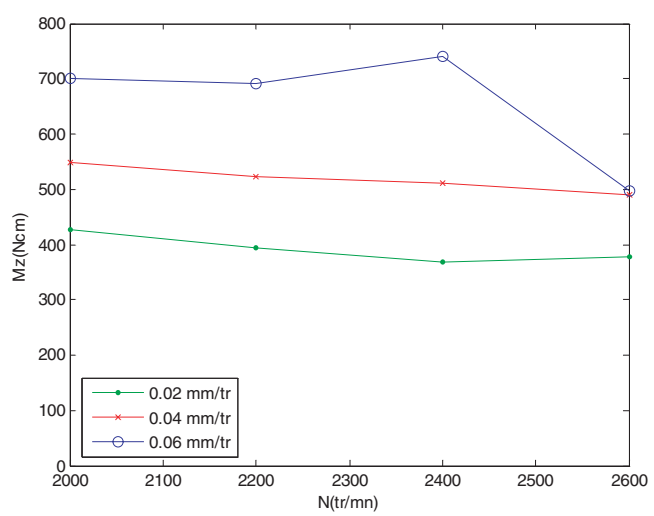

Fig. 14. Influence de la vitesse de rotation sur le couple dans le cas de l'usinage de l'aluminium.

l'aluminium que dans le carbone pour des paramètres de coupe identiques (2000 tr.min ${ }^{-1}, 0,04 \mathrm{~mm} . \mathrm{tr}^{-1}$ ).

\subsubsection{Conclusion}

Les paramètres opératoires non maîtrisés lors de l'usinage des panneaux de structures engendrent des poussées importantes (jusqu'à 400 N). La déformation du panneau laisse un espace libre entre les panneaux aluminium et composite qui risque d'être bouché par les copeaux. 


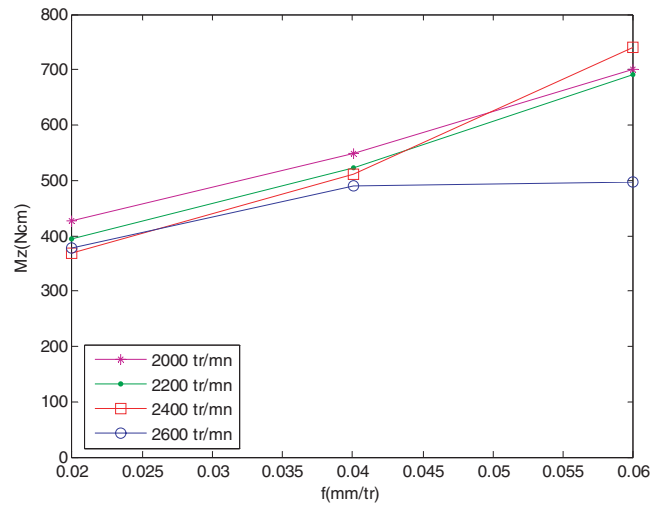

Fig. 15. Influence de l'avance sur le couple dans le cas de l'usinage de l'aluminium.

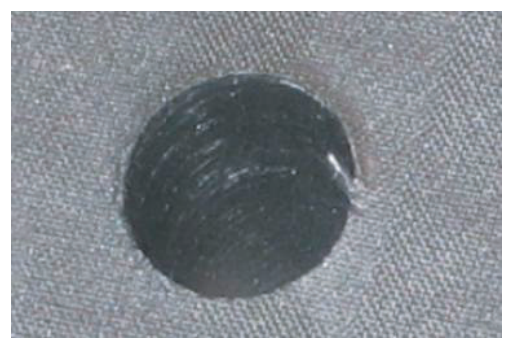

Fig. 16. Plis non coupés francs.

D'autre part, en cas d'utilisation d'une machine portative pour faire le perçage des panneaux, il est nécessaire de dimensionner la machine en fonction des couples et efforts mis en jeu. La minimisation des efforts permettra au système de rester « portatif ».

Cette première étude nous permet de prédimensionner une machine portative. Dans le carbone, la poussée maximale atteinte est de l'ordre de $250 \mathrm{~N}$. Afin de respecter cette poussée, on doit fixer une avance maximale dans l'aluminium autour de 0,03 mm.tr ${ }^{-1}$.

Cette avance crée un couple de 450 Ncm dans l'aluminium. On en déduit alors une avance dans le carbone de $0,13 \mathrm{~mm} \cdot \mathrm{tr}^{-1}$.

\subsection{Défauts macro et microgéométriques rencontrés}

Nous n'avons observé que deux types de défauts macrogéométriques sur les trois généralement relevés. Des plis non coupés francs sont observés en entrée de perçage dans le carbone (Fig. 16). Dans l'aluminium, des bavures importantes peuvent être réduites en minimisant l'avance à la sortie matière (Fig. 17). On remarque par contre qu'il n'y a absolument pas d'écaillage en sortie d'outil même avec les fortes avances.

Les premières mesures de qualité indiquent que l'état de surface se dégrade en fonction de l'augmentation de l'avance. Ce fait est certainement dû à la difficulté de l'évacuation du copeau.

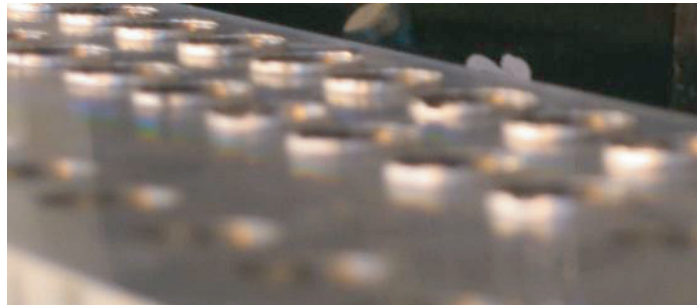

Fig. 17. Bavure dans l'aluminium.

\subsection{Conclusion et perspectives}

L'épaisseur des panneaux multi-matériaux est fonction de la raideur souhaitée. En conséquence, on ne peut prédire ni l'épaisseur à percer, ni la position de la zone de transition d'un matériau à un autre, il devient donc impossible de mettre en place une méthodologie de surveillance d'usinage classique. Une surveillance prédictive étant impossible, il faut envisager une surveillance adaptative. Cette étude montre donc qu'un suivi d'usinage peut être mis en place en adaptant les conditions de surveillances aux matériaux par une détection des seuils de transitions.

En outre, nous pouvons conclure de cette première étude que :

- La vitesse de coupe influence peu, dans le domaine étudié, sur le couple et l'effort de poussée.

- Les poussées et couples sont fonctions du matériau. En particulier pour les alliages d'aluminium, ces grandeurs sont jusqu'à deux à trois fois plus importantes que pour les composites carbones.

- Les efforts de poussée et les couples sont dépendants de la valeur d'avance.

- Il est impératif de faire varier indépendamment la fréquence de rotation et l'avance en fonction du matériau rencontré.

- Les transitions comme la débouchure sont détectables par les signaux d'effort et de couple.

Différentes voies pour la poursuite de ces travaux sont envisageables :

- la mise en place d'une méthodologie de surveillance adaptative;

- la définition de paramètres optimums de coupe dans le cycle de perçage afin de réduire le couple tout en améliorant l'état de surface;

- l'étude de l'influence de l'usure de l'outil (dégradation de l'acuité d'arête) sur la qualité microgéométrique des alésages.

Remerciements. Les auteurs remercient Frédéric LenOUVEL de SYNERVIA (2 avenue du professeur Jean Rouxel, 44475 Carquefou, France) pour son aide dans la réalisation des essais. 


\section{Références}

[1] B. Furet, B. Jolivel, D. Le Borgne, Les dernières avancées en usinage par fraisage et perçage des matériaux composites pour l'aéronautique, JEC Groups, 2005

[2] H. Ho-Cheng, C.K.H. Dharan, Delamination during drilling in composite laminates, ASME: J. Eng. Industry 112 (1990) 236-239

[3] F. Lachaud, R. Piquet, F. Collombet, L. Surcin, Drilling of composite structures, Composite Structures 52 (2001) $511-516$

[4] R. Zitoune, F. Collombet, R. Piquet, F. Lachaud, P. Pasquet, Experiment-calculation comparison of the cutting conditions representative of the long fibre composite drilling phase, Composites Science and Technology 65 (2005) 455-466

[5] D. Kim, M. Ramulu, Drilling process optimization for graphite/bismaleimide-titanium alloy stacks, Composite Structures 63 (2004) 101-114

[6] M. Ramulu, T. Branson, D. Kim, A study on the drilling of composite and titanium stacks, Composite Structures 54 (2001) 67-77

[7] E. Persson, I. Errikson, L. Zackrisson, Effects of hole machining defects on strength and fatigue life of composite laminates, Composites Part A28 (1997) 141-151

[8] P. Ghidossi, M. El Mansori, F. Pierron, Influence de la découpe d'éprouvettes en composite sur leur tenue mécanique, Mécanique \& Industries 3 (2002) 361-378 